\section{Mining HCV to sabotage HIV}

\section{By Michael J. Haas, Senior Writer}

HIV microbicides have been in development for at least a decade, but none has shown complete blocking of vaginal virus transmission. A major challenge has been identifying microbicidal agents whose actions are broad enough to minimize the potential for viral resistance and yet specific enough to kill HIV without irritating the vaginal mucosa-a side effect that can actually increase the risk of infection. ${ }^{1}$

Researchers at The Scripps Research Institute, VU University Medical Center in Amsterdam and Bioqual Inc. now have reported in the Proceedings of the National Academy of Sciences that a peptide derived from HCV-C5A-disrupts HIV's glycoprotein envelope and prevents the virus from infecting host cells. ${ }^{2}$

According to company researchers and an international product development partnership contacted by SciBX, further development of this promising peptide will hinge on issues such as solubility, dosage considerations and manufacturing.

The research team was led by Francis Chisari, professor in and head of the Department of Immunology and Microbial Science at Scripps, and Philippe Gallay, associate professor of immunology at Scripps. Microbicide expert and team member Brigitte Sanders-Beer, senior scientist at Bioqual, was formerly senior project leader of infectious disease research at Southern Research Institute.

C5A is an 18-residue, $\alpha$-helical peptide found within HCV's nonstructural protein $5 \mathrm{~A}$ (NS5A), which is believed to play a role in viral replication.

The researchers found that $\mathrm{C} 5 \mathrm{~A}$ had virucidal activity against a broad range of HIV isolates in vitro, killing the virus in a matter of minutes and preventing it from infecting all three of its host cell targets: $\mathrm{CD} 4^{+} \mathrm{T}$ cells, dendritic cells and macrophages.

The peptide blocked infection when used up to two hours before and after viral exposure. It offered no protection when used four hours prior to exposure.

The team also showed that $\mathrm{C} 5 \mathrm{~A}$ was not toxic to cervical epithelial cells at concentrations about 100 times greater than those required for the in vitro activity-an important observation because vaginal cytotoxicity and ensuing inflammation caused by a microbicide can potentially increase the risk of HIV infection.

Collectively, these results led the team to suggest that C5A could be the forerunner of a new generation of HIV microbicides.
The envelope, please

The paper comes on the heels of another PNAS study-also led by Chisari and Gallay and including researchers at Scripps and the University of Maryland-in which the team screened a library of overlapping 18-residue peptides, derived from the HCV polyprotein, for antiviral activity against $\mathrm{HCV}^{3}$

Peptides derived from functional domains of HIV previously have been shown to have activity against HIV, thanks to the availability of appropriate model systems. ${ }^{4,5}$ But the same had not been possible in the field of HCV research. Only recent technological advances and new in vitro models of $\mathrm{HCV}$ infection have finally paved the way to exploring the anti-HCV activity of HCV-derived peptides.

One peptide in Chisari and Gallay's screen exhibited far greater activity against $\mathrm{HCV}$ than any of the others. That peptide-which they dubbed C5A-also showed virucidal activity against a number of other viruses, including HIV, Dengue and West Nile.

"We expected to find activity of C5A against only HCV" in that broader study, Gallay told SciBX. "The activity against HIV was surprising because HIV has a different route of infection compared to HCV."

The anti-HIV activity led the team to its hypothesis about C5A's mechanism of action.

C5A is part of NS5A's anchor domain, which inserts itself into the lipid bilayer of a host cell's endoplasmic reticulum, as shown in a 2005 study by a research team at The Rockefeller University. ${ }^{6}$ The ChisariGallay team thinks C5A inserts itself into the glycoprotein envelope of HIV and HCV in a similar manner-but in those cases, it disrupts the envelope that each virus needs to invade host cells.

It might seem paradoxical that a viral protein domain contains something lethal to the virus itself. But it is important to understand that "C5A does not exist naturally," Gallay said. "C5A is only a small region of the complete HCV NS5A protein. Moreover, NS5A, in contrast to C5A, does not block HIV infection."

Incubation of HIV with trypsin-a human digestive enzyme that breaks down most proteins-did not prevent C5A from killing the virus, leading the team to conclude that $\mathrm{C} 5 \mathrm{~A}$ probably binds a lipid, not a protein, in the viral envelope.

"One of our next steps is to identify exactly what molecule the peptide binds," Gallay said. Once identified, C5A's target could be used to screen small molecules as potential new treatments against HIV infection, he said.

Susan Erickson-Viitanen, director of virology drug development at Incyte Corp., had several suggestions on how C5A's target could be identified.

"With three tryptophans in the peptide sequence, they can do some clever experiments to identify what C5A binds in the HIV envelope," she said.

For example, Erickson-Viitanen noted that $p$-benzoyl-L-phenylalanine (Bpa) - a photoreactive amino acid analog-can be substituted for tryptophan (Trp) without compromising a peptide's $\alpha$-helical 
structure. Bpa can be photoinduced to bind covalently to other molecules, and subsequent structural analysis can reveal what Bpa has bound, she said.

Bpa was first described in the literature by Erickson-Viitanen and colleagues at E.I. DuPont de Nemours and Co. in the 1980s, when they used Bpa to study binding of several peptides to the calciumbinding protein calmodulin. ${ }^{7}$

"If the researchers have some biophysical expertise, they can do a number of not-too-onerous experiments" to understand C5A's binding behavior-perhaps directed toward developing a smaller, more readily synthesized peptide, she said.

Gallay agreed C5A wasn't an optimum drug candidate.

"Once we identify what viral envelope molecule C5A binds, we could screen for drug-like molecules that could target it," he said. "It would be better to find something smaller than C5A-perhaps even a shorter peptide-because C5A is expensive to synthesize."

Going forward, Gallay said his group is pursuing C5A as both a microbicide to prevent HIV and as an HIV therapeutic.

Scripps, which holds the patent on C5A, has out-licensed the peptide to Viriome Inc., a company formed by Chisari to develop C5A as a treatment for $\mathrm{HIV}, \mathrm{HCV}$, dengue and other viruses against which the peptide has shown activity. Viriome is seeking commercial partners to advance C5A to the clinic, Chisari said.

Gallay said the researchers, in collaboration with Viriome, plan to test the serum-binding properties of a series of C5A derivatives, hop-

\section{"If they're right in how it works - that it is specific for HIV, HCV and some other viruses - and if it's not toxic to host cells, then they really have something here."}

-Al Profy,

Indevus Pharmaceuticals
That could mean either looking for microscopic signs of inflammation or looking at levels of proinflammatory cytokines in the vaginal cells.

"Part of development would require finding a cost-effective manufacturing process," he added. "This is especially significant for microbicides intended for use in developing countries."

Profy said the peptide also might encounter formulation difficulties-whether as a microbicide or an injectable-because it is unclear how water soluble it is, and microbicides are ideally water-based formulations. He noted that most of the experiments reported in the PNAS study were performed in dimethylsulfoxide, a solvent that can dissolve many drug-like compounds but is unsafe for use in humans.

Erickson-Viitanen cautioned that if the peptide is not sufficiently water soluble, it might not be possible to achieve an effective concentration for microbicidal use. "Even for a low molecular weight compound-which $\mathrm{C} 5 \mathrm{~A}$ isn't-the concentration required for a microbicide is huge relative to what is required" to show in vitro or in ivo activity, she said.

Additionally, Erickson-Viitanen said developing an 18-residue peptide into an injectable treatment for HIV had a low probability of success.

"Synthesis of an 18-mer requires a lot more time and effort than synthesizing a small molecule," she said. Such a synthesis would require at least 18 steps-each one involving work-up, purification and a commensurate reduction in the overall yield of the peptide. The synthesis of a drug-like molecule usually involves far fewer steps, she said.

Erickson-Viitanen was also concerned that ing to find one that will not be inactivated in serum, and thus make a better candidate for an injectable HIV treatment.

\section{Peptide microbicide}

Representatives of companies and an international nonprofit HIV drug development organization contacted by SciBX agreed that C5A holds promise as a microbicide, with the next steps being to address potential cytotoxicity and formulation issues.

"The data in the paper look encouraging," said Al Profy, VP of preclinical development at Indevus Pharmaceuticals Inc. "It's an interesting molecule, and if they're right in how it works-that it is specific for HIV, HCV and some other viruses-and if it's not toxic to host cells, then they really have something here."

Indevus' PRO 2000, a naphthalene sulfate polymer gel that binds HIV gp120, is in two Phase III trials to prevent HIV infection. The trials are scheduled to finish in mid-2008 and 2009. The company also has PRO 2000 in a Phase II/IIb trial with ReProtect Inc.'s BufferGel, a broad-spectrum, acidifying polymer gel. That trial, also to prevent HIV infection, is expected to finish in late 2008.

To advance C5A, Profy said additional cytotoxicity experiments were needed because vaginal microbicides usually require a larger dose of antiviral agent than oral formulations to be effective. Thus the therapeutic window between an effective and toxic dose could be quite narrow, he said.

"As development of this peptide proceeds, they will have to study the effect of dosing on vaginal irritation in animal models," said Profy.
C5A lost its in vitro potency when added to cells four hours before the cells were exposed to HIV. "It is not clear whether this is physical instability - a physical limitation of the peptide-or something else to do with the experiment itself," she said.

The researchers still don't know why the peptide becomes inactive when given four hours prior to infection, Gallay said. "We know that it is not degraded, but it's probably bound to a protein" in the serumbased cell culture medium, he said. "We have not tested the resistance of C5A in vaginal fluids yet."

A microbicide that could not be used more than two hours before sexual intercourse would not be very practical, especially in the developing world, said Erickson-Viitanen. "You don't want a microbicide that requires such a precise timing of use," she said.

In March, Incyte halted development of INCB9471, a CC chemokine receptor 5 (CCR5) antagonist that was the company's only HIV therapeutic, because of the high cost, time and labor requirements of the program. Incyte said it would look to out-license the compound instead of beginning Phase IIb trials.

Joseph Romano, executive director for research and development at the International Partnership for Microbicides (IPM), thought C5A was an interesting candidate but said it was too early to say whether it could be developed as a microbicide.

IPM is a nonprofit product development partnership (PDP) established in 2002 to prevent the spread of HIV by accelerating the development and availability of a safe and effective microbicide for use by women in developing countries. 


\section{TARGETS \& MECHANISMS}

"This peptide is interesting because it appears to have a unique mode of action, compared to other compounds in development as microbicides that interfere with viral attachment, fusion, entry and replication processes," Romano said. "The peptide is also attractive because this interference happens quite early in the infection process. We like drugs that act earlier rather than later."

But he concurred with Profy and Erickson-Viitanen on the obstacles of developing microbicidal peptides, including the facts that they are expensive to make and that their aqueous stability is sometimes suspect, presenting formulation difficulties.

"Also, peptides don't lend themselves to delivery with a vaginal ring, the way small molecules like tenofovir and maraviroc do," he said.

Gilead Sciences Inc. markets the reverse transcriptase inhibitor Viread tenofovir in the EU and U.S. to treat HIV infection. Pfizer Inc. markets maraviroc, a CCR5 antagonist, as Celsentri in the EU and Selzentry in the U.S. to treat HIV. IPM received royalty-free licenses to develop tenofovir and maraviroc as microbicides from Gilead in 2006 and Pfizer in February, respectively.

Nevertheless, Romano said IPM doesn't discount peptides as potential microbicides, noting that in March, Merck \& Co. Inc. granted the PDP a royalty-free license to develop L'644, a fusion inhibitor targeting HIV gp41, as a microbicide.

"Right now we don't yet have an effective microbicide, so we have to be open to possibilities-even when a compound presents technical challenges to development," he said.

\section{The roads ahead}

Solubility and formulation issues aside, the good news is that C5A could be less resistance-prone than other HIV targets.

"It would be hard for HIV to develop resistance to the peptide," said Gallay. "In order to do so, the virus would have to generate its envelope" without the target molecule C5A binds, or the target molecule would have to mutate-which a lipid could not do as readily as a protein.

Nevertheless, any injectable HIV drug could face an uphill battle in the marketplace. The majority of HIV drugs are oral, and Fuzeon enfuvirtide, a first-in-class subcutaneous injectable for HIV from Trimeris Inc. and Roche, has had disappointing uptake. ${ }^{8}$

"It's a problem to be an injectable when there are so many options that are not," noted Erickson-Viitanen.
Progress toward the development of HIV microbicides also depends on the availability of good model systems for preclinical evaluation. The recent report by J. Victor GarciaMartinez, a professor of internal medicine at the University of Texas Southwestern Medical School, and colleagues, describing the use of humanized bone marrow-liver-thymus (BLT) mice as a model for intravaginal HIV infection represents an advance in this direction. ${ }^{9}$

"We are also collaborating with Victor Garcia to develop microbicides-first in Victor's mouse models, then eventually in the macaque model of vaginal transmission," Gallay said, both of which will include further toxicological studies.

\section{REFERENCES}

1. Haas, M., SciBX 1(3), 1-4; Feb. 14, 2008

2. Bobardt, M. et al. Proc. Natl. Acad. Sci. USA; published online March 31, 2008; doi:10.1073/pnas.0801388105

Contact: Francis V. Chisari, The Scripps Research Institute, La Jolla, Calif.

e-mail: fchisari@scripps.edu

Contact: Philippe Gallay, same affiliation as above e-mail: gallay@scripps.edu

3. Cheng, G. et al. Proc. Natl. Acad. Sci. USA 105, 3088-3093 (2008)

4. Lambert, D. et al. Proc. Natl. Acad. Sci. USA 93, 2186-2191 (1996)

5. Kilby, J. et al. Nat. Med. 4, 1302-1307 (1998)

6. Tellinghuisen, T. et al. Nature 453, 374-377 (2005)

7. Kauer, J. et al. J. Biol. Chem. 261, 10695-10700 (1986)

8. Lawrence, S. BioCentury 15(13), A20; March 19, 2007

9. Denton, P.W. et al. PLoS Med. 5, e16; published online January 15, 2008; doi:10.1371/journal.pmed.0050016

COMPANIES AND INSTITUTIONS MENTIONED

Bioqual Inc. (Pink:BIOQ), Rockville, Md.

E.I. du Pont de Nemours and Co. (NYSE:DD), Wilmington, Del. Gilead Sciences Inc. (NASDAQ:GILD), Foster City, Calif. Incyte Corp. (NASDAQ:INCY), Wilmington, Del. Indevus Pharmaceuticals Inc. (NASDAQ:IDEV), Lexington, Mass. International Partnership for Microbicides, Silver Spring, Md.

Merck \& Co. Inc. (NYSE:MRK), Whitehouse Station, N.J.

Pfizer Inc. (NYSE:PFE), New York, N.Y.

ReProtect Inc., Baltimore, Md.

Roche (SWX:ROG), Basel, Switzerland

The Rockefeller University, New York, N.Y.

The Scripps Research Institute, La Jolla, Calif.

Southern Research Institute, Frederick, Md.

Trimeris Inc. (NASDAQ:TRMS), Morrisville, N.C.

University of Texas Southwestern Medical School, Dallas, Texas

University of Maryland, College Park, Md.

Viriome Inc., West Chester, Pa.

VU University Medical Center, Amsterdam, the Netherlands 